\title{
A NOTE ON CONCENTRATION FOR BLOWUP SOLUTIONS TO SUPERCRITICAL SCHRÖDINGER EQUATIONS
}

\author{
QING GUO
}

(Communicated by James E. Colliander)

\begin{abstract}
We study the blowup dynamics of solutions to the $L^{2}$-supercritical nonlinear Schrödinger equation and prove that the blowup solution with bounded $\dot{H}^{s_{c}}$ norm must concentrate at least a fixed amount of the $\dot{H}^{s_{c}}$ norm and, also, its $L^{p_{c}}$ norm must concentrate at least a fixed $L^{p_{c}}$ norm. We show these properties without any further symmetry assumptions on the solution and partly generalize the results obtained in papers of Holmer and Roudenko and of Zhu, which only deal with the radially symmetric case. Our proof is based on the profile decomposition theorems.
\end{abstract}

\section{INTRODUCTION}

In this paper, we study several concentration properties of the blowup solutions to the following $L^{2}$-supercritical and $\dot{H}^{1}$-subcritical nonlinear Schrödinger equation:

$$
\left\{\begin{aligned}
i u_{t}+\Delta u+|u|^{p-1} u & =0, \quad(x, t) \in \mathbb{R}^{N} \times \mathbb{R}, \\
u(x, 0) & =u_{0}(x),
\end{aligned}\right.
$$

where $\frac{4}{N}+1<p<\frac{4}{N-2}+1$.

By scaling, equation (1.1) is $\dot{H}^{s_{c}}$-critical with $s_{c}=\frac{N}{2}-\frac{2}{p-1}$. As is well-known, for $s_{c}<0$, the equation is $L^{2}$-subcritical and all $H^{1}$ solutions are global. The smallest power for which blowup may occur is $p=\frac{4}{N}+1$, which is referred to as the $L^{2}$-critical case corresponding to $s_{c}=0$. For this case, the blowup theory is mainly connected to the notion of the ground state: the unique positive radial solution of the elliptic problem $\Delta Q-Q+|Q|^{\frac{4}{N}} Q=0$. There is abundant literature devoted to the study of the blowup mechanism (see [3, 15]), and it has been shown that there is a minimal amount of concentration of the $L^{2}$ norm (see [11, 14, 17]).

For the $\dot{H}^{1}$-critical case with $s_{c}=1$, the authors in [12] and [13] showed that any blowup solution with bounded kinetic energy must concentrate at least the kinetic energy of the ground state in the cases of dimensions $N=3,4,5$ (for radially symmetric initial data) and $N \geq 5$ respectively.

Received by the editors May 3, 2011 and, in revised form, November 8, 2011 and January 8, 2012 .

2010 Mathematics Subject Classification. Primary 35Q55, 35A15; Secondary 35B30.

Key words and phrases. Schrödinger equation, blowup solution, concentration, supercritical. 
In this paper, we are concerned with the $L^{2}$-supercritical and $\dot{H}^{1}$-subcritical case, i.e., $0<s_{c}<1$. In this case, Ginibre and Velo [6] established the local wellposedness results in the energy space $H^{1}$, while Cazenave and Weissler [4] did that in space $\dot{H}^{s_{c}} \cap \dot{H}^{1}$. More precisely, setting $2^{*}=\frac{2 N}{N-2}$, we have the following two propositions.

Proposition $1.1([6])$. Let $1<p<2^{*}-1$ and $u_{0} \in H^{1}$. Then, there exist some time $T_{+}>0$ and a unique solution $u(x, t) \in C\left(\left[0, T_{+}\right) ; H^{1}\right)$ to equation (1.1) such that either $T_{+}=\infty$ or $T_{+}<\infty$ and $\lim _{t \rightarrow T_{+}}\|u(t, x)\|_{H^{1}}=\infty$. Moreover, $u$ admits the following conservation laws in space $H^{1}: M(u)(t) \equiv \int|u(x, t)|^{2} d x=M\left(u_{0}\right)$ and

$$
\text { Energy : } \quad E(u)(t) \equiv \frac{1}{2} \int|\nabla u(x, t)|^{2} d x-\frac{1}{p+1} \int|u(x, t)|^{p+1} d x=E\left(u_{0}\right) .
$$

Proposition $1.2([4])$. Let $1<p<2^{*}-1$ and $u_{0} \in \dot{H}^{s_{c}} \cap \dot{H}^{1}$. Then, there exist some time $T_{+}>0$ and a unique solution $u(x, t) \in C\left(\left[0, T_{+}\right) ; \dot{H}^{s_{c}} \cap \dot{H}^{1}\right)$ to equation (1.1) such that either $T_{+}=\infty$ or $T_{+}<\infty$ and $\lim _{t \rightarrow T_{+}}\|u(t, x)\|_{\dot{H}^{1}}=\infty$. Moreover, $u$ satisfies the energy conservation law (1.2).

For the 3D cubic nonlinear Schrödinger equation with $s_{c}=\frac{1}{2}$ and $p=3$, there have been several results on both scattering and blowup solutions to (1.1), for which one can refer to $[8,9,10$. The author in 19 , has extended the scattering results to the general case $0<s_{c}<1$, while its blowup theory was also studied in [7]. In the present paper, we further study the dynamical properties of blowup solutions in such a general case. More precisely, applying profile decomposition theorems and using the variational structure associated to the corresponding elliptic problems, we prove that the blowup solution with bounded $\dot{H}^{s_{c}}$ norm must concentrate at least a fixed amount of $\dot{H}^{s_{c}}$ norm and its $L^{p_{c}}$ norm must concentrate at least a fixed $L^{p_{c}}$ norm as well (see Theorem 1.3 and Theorem 1.4 below). Following the idea from Keraani [11] about the $L^{2}$-critical case, our proof is based on the compactness argument without any further symmetric assumptions on the solution and, thus, has partly generalized the results obtained in [9, 20], which only dealt with the radially symmetric case.

In this paper, we denote the Sobolev space $H^{1}\left(\mathbb{R}^{N}\right)$ as $H^{1}$ and the space $L^{p}\left(\mathbb{R}^{N}\right)$ as $L^{p}$ with their norms denoted by $\|\cdot\|_{H^{1}}$ and $\|\cdot\|_{p}$ respectively for short. We define the Fourier transform on $\mathbb{R}^{N}$ by $\hat{f}(\xi)=(2 \pi)^{-N / 2} \int e^{-i x \xi} f(x) d x$. For $s \in$ $\mathbb{R}$, the pseudo-differential operator $(-\Delta)^{s}$ is defined by $\widehat{\left(\widehat{-\Delta)^{s}}\right.} f(\xi) \equiv|\xi|^{2 s} \hat{f}(\xi)$, which in turn defines the homogeneous Sobolev space $\dot{H}^{s}=\dot{H}^{s}\left(\mathbb{R}^{N}\right): \dot{H}^{s} \equiv$ $\left\{f \in \mathcal{S}^{\prime}\left(\mathbb{R}^{N}\right): \int|\xi|^{2 s}|\hat{f}(\xi)|^{2} d \xi<\infty\right\}$ with its norm defined by $\|f\|_{\dot{H}^{s}}=\left\|(-\Delta)^{\frac{s}{2}} f\right\|_{2}$, where $\mathcal{S}^{\prime}\left(\mathbb{R}^{N}\right)$ denotes the space of tempered distributions. For convenience, we use the notation $C$ standing for variant absolute constants and introduce the following notation: $s_{c}=\frac{N}{2}-\frac{2}{p-1}, p_{c}=\frac{2 N}{N-2 s_{c}}=\frac{N(p-1)}{2}, 2^{*}=\frac{2 N}{N-2}$. Note that, by Sobolev imbeddings, we have $\dot{H}^{1} \hookrightarrow L^{2^{*}}$ and $\dot{H}^{s_{c}} \hookrightarrow L^{p_{c}}$.

The main results obtained in this paper are as follows:

Theorem 1.3. Let $u_{0} \in \dot{H}^{s_{c}} \cap \dot{H}^{1}$ be such that the corresponding solution $u$ to (1.1) blows up in finite time $T_{+}>0$ satisfying

$$
\sup _{t \in\left[0, T_{+}\right)}\|u(t)\|_{\dot{H}^{s_{c}}}<\infty .
$$


Assume that $\lambda(t)>0$ such that

$$
\lambda(t)\|\nabla u(t)\|_{2}^{\frac{1}{1-s_{c}}} \rightarrow \infty,
$$

as $t \rightarrow T_{+}$. Then, there exists $x(t) \in \mathbb{R}^{N}$ such that

$$
\liminf _{t \rightarrow T_{+}} \int_{|x-x(t)| \leq \lambda(t)}\left|(-\Delta)^{\frac{s_{c}}{2}} u(t, x)\right|^{2} d x \geq\|\tilde{Q}\|_{\dot{H}^{s_{c}}}^{2},
$$

where $\tilde{Q}$ solves the elliptic equation

$$
-\Delta Q+\frac{p-1}{2}(-\Delta)^{s_{c}} Q-|Q|^{p-1} Q=0 .
$$

Theorem 1.4. Let $u_{0} \in \dot{H}^{s_{c}} \cap \dot{H}^{1}$ be such that the corresponding solution $u$ to (1.1) blows up in finite time $T_{+}>0$ and satisfies (1.3). Assume that $\lambda(t)>0$ satisfying (1.4). Then, there exists $x(t) \in \mathbb{R}^{N}$ such that

$$
\liminf _{t \rightarrow T_{+}} \int_{|x-x(t)| \leq \lambda(t)}|u(t, x)|^{p_{c}} d x \geq\left\|Q^{\prime}\right\|_{p_{c}}^{p_{c}},
$$

where $Q^{\prime}$ solves the elliptic equation

$$
-\Delta Q+|Q|^{p_{c}-2} Q-|Q|^{p-1} Q=0 .
$$

Remark 1.5. In view of Proposition 2.3 to be introduced in the following section, the $\dot{H}^{s_{c}}$ norm bound assumption (1.3) should, in fact, be equivalent to $\|\tilde{Q}\|_{\dot{H}^{s_{c}}} \leq$ $\sup _{t \in\left[0, T_{+}\right)}\|u(t)\|_{\dot{H}^{s_{c}}}<\infty$ with the same $\tilde{Q}$ as in Theorem 1.3 .

The paper is structured as follows. We prove Theorems 1.3 and 1.4 in section 3, which follows a review of some variational facts related to some elliptic problems in section 2. In the last section of this paper, we give some remarks on the divergent solutions to (1.1) obtained in [7] and describe the corresponding concentration properties as $t \rightarrow+\infty$, which, to the author's knowledge, are new.

\section{Some Variational estimates}

In this section, we review some variational facts related to the corresponding elliptic equations which play an important role in our main theorems.

From [7], it is known that the sharp constant $c_{G N}$ in the Gagliardo-Nirenberg inequality

$$
\|u\|_{p+1}^{p+1} \leq c_{G N}\|\nabla u\|_{2}^{\frac{N(p-1)}{2}}\|u\|_{2}^{2-\frac{(N-2)(p-1)}{2}}
$$

is attained by the function $Q$, which is the ground state of the elliptic equation $-\left(1-s_{c}\right) Q+\Delta Q+|Q|^{p-1} Q=0$, and $c_{G N}$ can be expressed by $c_{G N}=$ $\|Q\|_{p+1}^{p+1} /\|\nabla Q\|_{2}^{\frac{N(p-1)}{2}}\|Q\|_{2}^{2-\frac{(N-2)(p-1)}{2}}$.

Using the same method as in [18, we can obtain the following theorem, a variational structure to study the $L^{2}$-supercritical problem (1.1).

Theorem 2.1. Let $p_{c}-1<p<2^{*}-1$. The best constant $c_{p_{c}}$ in

$$
\|f\|_{p+1}^{p+1} \leq c_{p_{c}}\|f\|_{p_{c}}^{p-1}\|\nabla f\|_{2}^{2}, \quad f \in \dot{H}^{1} \cap L^{p_{c}},
$$

is attained at $c_{p_{c}}=\frac{p+1}{2}\left\|Q^{\prime}\right\|_{p_{c}}^{-(p-1)}$, where $Q^{\prime}$ solves the elliptic equation (1.8). 
Proof. It suffices to show that

$$
\inf _{u \in\left(\dot{H}^{1} \cap L^{p_{c}}\right) \backslash\{0\}} J(u)=\frac{2}{p+1}\left\|Q^{\prime}\right\|_{p_{c}}^{p-1}, \quad \text { where } \quad J(u)=\frac{\|u\|_{p_{c}}^{p-1}\|\nabla u\|_{2}^{2}}{\|u\|_{p+1}^{p+1}} .
$$

We set $J=\inf _{u \in\left(\dot{H}^{1} \cap L^{p_{c}}\right) \backslash\{0\}} J(u)$ and consider a minimizing sequence $\left\{v_{n}\right\}_{n=1}^{\infty}$. By inequality (2.2), we observe that $J>0$. Now, we rescale $\left\{v_{n}\right\}_{n=1}^{\infty}$ by setting $u_{n}(x)=\mu_{n} v_{n}\left(\lambda_{n}\right)$, where

$$
\lambda_{n}=\left(\left\|v_{n}\right\|_{p_{c}} /\left\|\nabla v_{n}\right\|_{2}\right)^{\frac{1}{1-s_{c}}}, \quad \mu_{n}=\left(\left\|v_{n}\right\|_{p_{c}}^{\frac{N}{2}-1} /\left\|\nabla v_{n}\right\|_{2}^{\frac{N}{2}-s_{c}}\right)^{\frac{1}{1-s_{c}}}
$$

so that $\left\|u_{n}\right\|_{p_{c}}=\left\|\nabla u_{n}\right\|_{2}=1$. Thus, as $n \rightarrow \infty$, we have $\left\|u_{n}\right\|_{p+1}^{-(p+1)}=J\left(u_{n}\right) \rightarrow$ $J>0$.

According to Berestychi and Lions (2), if $f_{n}$ is a minimizing sequence, then so is its Schwarz symmetrization. Thus, we may assume that $\left\{u_{n}\right\}_{n=1}^{\infty}$ is radially symmetric. Since $p_{c}<p+1<2^{*}$, there exist a subsequence (still denoted by itself) and $u \in \dot{H}^{1} \cap L^{p_{c}}$ such that $u_{n} \rightarrow u$ weakly in $\dot{H}^{1} \cap L^{p_{c}}$ and $u_{n} \rightarrow u$ strongly in $L^{p+1}$. Then we get from the definition of $J$ that

$$
J \leq J(u)=\frac{\|u\|_{p_{c}}^{p-1}\|\nabla u\|_{2}^{2}}{\|u\|_{p+1}^{p+1}} \leq \frac{\lim _{n \rightarrow \infty}\left\|u_{n}\right\|_{p_{c}}^{p-1} \lim _{n \rightarrow \infty}\left\|\nabla u_{n}\right\|_{2}^{2}}{\|u\|_{p+1}^{p+1}}=\lim _{n \rightarrow \infty} J\left(u_{n}\right)=J,
$$

which implies that $J(u)=J$ and $\|u\|_{p_{c}}^{p-1}\|\nabla u\|_{2}^{2}=\lim _{n \rightarrow \infty}\left\|u_{n}\right\|_{p_{c}}^{p-1} \lim _{n \rightarrow \infty}\left\|\nabla u_{n}\right\|_{2}^{2}$ $=1$ by construction. Since $0<\|u\|_{p_{c}}^{p-1},\|\nabla u\|_{2}^{2} \leq 1$, we get from the above equality that $\|u\|_{p_{c}}=\|\nabla u\|_{2}=1$. From $J(u)=J$, we obtain that $\left.\frac{d}{d t} J(u+t w)\right|_{t=0}=0$ for all $w \in \dot{H}^{1} \cap L^{p_{c}}$, and so we obtain that $-\Delta u+\frac{p-1}{2}|u|^{p_{c}-2} u-\frac{J(p+1)}{2}|u|^{p-1} u=0$. Now let $Q^{\prime}$ be defined by $u(x)=a Q^{\prime}(b x)$ with

$$
a=\left(\frac{(p+1) J}{p-1}\right)^{\frac{N}{N p_{c}-2 p_{c}-2 N}}, \quad b=\left(\frac{p-1}{2}\right)^{\frac{1}{2}}\left(\frac{(p+1) J}{p-1}\right)^{\frac{N p_{c}-2 N}{N p_{c}-2 p_{c}-2 N}}
$$

so that $Q^{\prime}$ is a solution of (1.8) and that $\left\|Q^{\prime}\right\|_{p_{c}}^{p_{c}}=a^{-p_{c}} b^{N}\|u\|_{p_{c}}^{p_{c}}=a^{-p_{c}} b^{N}=$ $\left(\frac{p+1}{2}\right)^{\frac{N}{2}} J^{\frac{N}{2}}$. That is, $J=\frac{2}{p+1}\left\|Q^{\prime}\right\|_{p_{c}}^{p-1}$, and we complete the proof of Theorem 2.1.

As a consequence of (2.2), we can obtain a global well-posedness result.

Theorem 2.2. Let $1<p<2^{*}-1$. Assume that $u_{0} \in \dot{H}^{s_{c}} \cap \dot{H}^{1}$ and $u(t)$ is the corresponding solution to (1.1) such that $\sup _{t}\|u(t)\|_{p_{c}}<\left\|Q^{\prime}\right\|_{p_{c}}$ with $Q^{\prime}$ the solution of the elliptic equation (1.8). Then $u(t)$ exists globally in time.

Proof. By (2.2) and the conservation of energy, we immediately get

$$
E\left(u_{0}\right)=E(u)=\frac{1}{2}\|\nabla u\|_{2}^{2}-\frac{1}{p+1}\|u\|_{p+1}^{p+1} \geq \frac{1}{2}\left(1-\left(\frac{\|u\|_{p_{c}}}{\left\|Q^{\prime}\right\|_{p_{c}}}\right)^{p-1}\right)\|\nabla u\|_{2}^{2},
$$

which combined with the assumption $\sup _{t}\|u(t)\|_{p_{c}}<\left\|Q^{\prime}\right\|_{p_{c}}$ implies that $\|\nabla u(t)\|_{2}$ is bounded for all $t$. In view of the well-posedness results (Proposition 1.2), we obtain that the solution of (1.1) must be global.

The same method can be used by showing another global existence result: 
Proposition 2.3. Let $1<p<2^{*}-1$. Assume that $u_{0} \in \dot{H}^{s_{c}} \cap \dot{H}^{1}$ and $u(t)$ is the corresponding solution to (1.1) such that $\sup _{t}\|u(t)\|_{\dot{H}^{s_{c}}}<\|\tilde{Q}\|_{\dot{H}^{s_{c}}}$, with $\tilde{Q}$ the solution of the elliptic equation (1.6). Then, $u(t)$ exists globally in time.

Similarly to Theorem 2.2 , to show this proposition we just need another sharp Gagliardo-Nirenberg inequality

$$
\|u\|_{p+1}^{p+1} \leq c_{G}\|u\|_{\dot{H}^{s_{c}}}^{p-1}\|u\|_{\dot{H}^{1}}^{2}, \quad u \in \dot{H}^{s_{c}} \cap \dot{H}^{1},
$$

with the best constant $c_{G}=\frac{p+1}{2\|\tilde{Q}\|_{H^{s} s_{c}}^{p-1}}$, where $\tilde{Q}$ solves the elliptic equation (1.6). In fact, following the idea in [18] again, this result can be obtained by the same method as used in the proof of Theorem 2.1, which we will not repeat here. One can also find the result in $[20$.

Finally, we review some facts about the critical elliptic equation

$$
\Delta W+|W|^{\frac{4}{N-2}} W=0
$$

By the work of Aubin [1] and Talenti [16, we have the following characterization of $W(x)$, which is the radially symmetric and decreasing solution to (2.4):

$$
\|u\|_{2^{*}} \leq c_{N}\|\nabla u\|_{2}, \quad u \in \dot{H}^{1} .
$$

Moreover, the equality holds if and only if $u(x)=e^{i \theta} \lambda^{\frac{N-2}{2}} W\left(\lambda\left(x-x_{0}\right)\right)$ for some $\left(\theta, \lambda, x_{0}\right) \in[-\pi, \pi] \times \mathbb{R}_{*}^{+} \times \mathbb{R}^{N}$, where $\mathbb{R}_{*}^{+}=\mathbb{R}^{+} \backslash\{0\}$. The best constant $c_{N}$ can be described as $c_{N}^{N}\|\nabla W\|_{2}^{2}=1$.

Remark 2.4. By the interpolation inequality of the space $L^{p}$ and the Sobolev imbedding (2.5), we have $\|u\|_{p+1}^{p+1} \leq\|u\|_{p_{c}}^{p-1}\|u\|_{2^{*}}^{2} \leq c_{N}^{2}\|u\|_{p_{c}}^{p-1}\|\nabla u\|_{2}^{2}$, which immediately implies that $c_{p_{c}} \leq c_{N}^{2}$.

\section{Proof of the Main Results}

The main tool we used to prove Theorem 1.3 and Theorem 1.4 is the following proposition.

Proposition 3.1. Let $\left\{v_{n}\right\}_{n=1}^{\infty}$ be a bounded family of $\dot{H}^{s_{c}} \cap \dot{H}^{1}$. Then there exist a subsequence of $\left\{v_{n}\right\}_{n=1}^{\infty}$ (still denoted by itself), a family of sequences $\left\{x_{n}^{j}\right\} \subset \mathbb{R}^{N}$, and a sequence of functions $\left\{V^{j}\right\}_{j=1}^{\infty} \subset \dot{H}^{s_{c}} \cap \dot{H}^{1}$ such that:

i) There holds the following asymptotic pairwise divergence as $n \rightarrow \infty$ :

$$
\forall k \neq j, \quad\left|x_{n}^{k}-x_{n}^{j}\right| \rightarrow \infty .
$$

ii) For every $l \geq 1$ and every $x \in \mathbb{R}^{N}$, we have

$$
v_{n}(x)=\sum_{j=1}^{l} V^{j}\left(x-x_{n}^{j}\right)+v_{n}^{l}(x)
$$

with $\lim \sup _{n \rightarrow \infty}\left\|v_{n}^{l}\right\|_{r} \rightarrow 0$ as $l \rightarrow \infty$ for every $r \in\left(p_{c}, 2^{*}\right)$. Moreover,

$$
\left\|v_{n}\right\|_{\dot{H}^{s}}^{2}=\sum_{j=1}^{l}\left\|V^{j}\right\|_{\dot{H}^{s}}^{2}+\left\|v_{n}^{l}\right\|_{\dot{H}^{s}}^{2}+o(1)
$$

for any $s \in\left[s_{c}, 1\right]$, where $o(1)=o_{n}(1) \rightarrow 0$ as $n \rightarrow \infty$. 
The proof of Proposition 3.1 is similar to a well-known result from [11], where the author considered the $H^{1}$-bounded sequence. In fact, it is essentially because $H^{1}$ is no other than $\dot{H}^{\tilde{s}_{c}} \cap \dot{H}^{1}$ with $\tilde{s}_{c}=0$. In [20] there was a similar argument, but to be self-contained, we now sketch the proof of Proposition 3.1

Proof. Let $\mathcal{V}\left(v_{n}\right)$ be the set of functions obtained as weak limits of subsequences of the translated $v_{n}\left(\cdot+x_{n}\right)$ with $\left\{x_{n}\right\}_{n=1}^{\infty} \subset \mathbb{R}^{N}$. We denote $\eta\left(v_{n}\right)=\sup \left\{\left(\|\nabla v\|_{2}^{2}+\right.\right.$ $\left.\left.\|v\|_{\dot{H}^{s_{c}}}^{2}\right)^{\frac{1}{2}}: v \in \mathcal{V}\left(v_{n}\right)\right\}$. Clearly, $\eta\left(v_{n}\right) \leq \lim \sup _{n \rightarrow \infty}\left(\left\|v_{n}\right\|_{\dot{H}^{s_{c}}}^{2}+\left\|v_{n}\right\|_{\dot{H}^{1}}^{2}\right)^{\frac{1}{2}}$. We claim that there exist a sequence $\left\{V^{j}\right\}_{j=1}^{\infty}$ of $\mathcal{V}\left(v_{n}\right)$ and a family of sequences $\left\{x_{n}^{j}\right\} \subset \mathbb{R}^{N}$ such that for any $k \neq j,\left|x_{n}^{k}-x_{n}^{j}\right| \rightarrow \infty$ as $n \rightarrow \infty$, and up to a subsequence,

$$
v_{n}(x)=\sum_{j=1}^{l} V^{j}\left(x-x_{n}^{j}\right)+v_{n}^{l}(x), \quad \eta\left(v_{n}^{l}\right) \rightarrow 0 \quad \text { as } l \rightarrow \infty
$$

such that (3.3) holds. Indeed, if $\eta\left(v_{n}\right)=0$, we can take $V^{j} \equiv 0$ for all $j$. Otherwise, we choose $V^{1} \in \mathcal{V}\left(v_{n}\right)$ such that $\left(\left\|V^{1}\right\|_{\dot{H}^{s_{c}}}^{2}+\left\|V^{1}\right\|_{\dot{H}^{1}}^{2}\right)^{\frac{1}{2}} \geq \frac{1}{2} \eta\left(v_{n}\right)>0$. By definition, there exists some sequence $\left\{x_{n}^{1}\right\} \subset \mathbb{R}^{N}$ such that, up to a subsequence, $v_{n}\left(\cdot+x_{n}^{1}\right) \rightarrow$ $V^{1}$ weakly in $\dot{H}^{s_{c}} \cap \dot{H}^{1}$. Setting $v_{n}^{1}(x) \equiv v_{n}(x)-V^{1}\left(x-x_{n}^{1}\right)$, we get $v_{n}^{1}\left(\cdot+x_{n}^{1}\right) \rightarrow 0$, and then

$$
\left\|v_{n}\right\|_{\dot{H}^{s_{c}}}^{2}=\left\|V^{1}\right\|_{\dot{H}^{s_{c}}}^{2}+\left\|v_{n}^{1}\right\|_{\dot{H}^{s_{c}}}^{2}+o(1), \quad\left\|v_{n}\right\|_{\dot{H}^{1}}^{2}=\left\|V^{1}\right\|_{\dot{H}^{1}}^{2}+\left\|v_{n}^{1}\right\|_{\dot{H}^{1}}^{2}+o(1) .
$$

It is known that for any $s \in\left(s_{c}, 2^{*}\right), \dot{H}^{s}$ is an interpolation space between $\dot{H}^{s_{c}}$ and $\dot{H}^{1}$, and the square of its norm $\|\cdot\|_{\dot{H}^{s}}^{2}$ is equivalent to $\|\cdot\|_{\dot{H}^{s_{c}}}^{2}+\|\cdot\|_{\dot{H}^{1}}^{2}$, which by (3.5) implies (3.3) in the case $l=1$. Now, we replace $v_{n}$ by $v_{n}^{1}$ and repeat the same process. If $\eta\left(v_{n}^{1}\right)>0$ we get $V^{2}>0, x_{n}^{2}$ and $v_{n}^{2}$. Moreover, we can obtain $\left|x_{n}^{1}-x_{n}^{2}\right| \rightarrow \infty$ as $n \rightarrow \infty$. Otherwise, if up to a subsequence, $x_{n}^{1}-x_{n}^{2} \rightarrow x_{0}$ for some $x_{0} \in \mathbb{R}^{N}$, then since $v_{n}^{2}\left(x+x_{n}^{2}\right)=v_{n}^{1}\left(x+x_{n}^{2}\right)-V^{2}(x)=v_{n}^{1}\left(x+\left(x_{n}^{2}-x_{n}^{1}\right)+\right.$ $\left.x_{n}^{1}\right)-V^{2}(x)$ and $v_{n}^{1}\left(\cdot+x_{n}^{1}\right) \rightarrow 0$, we get $V^{2}=0$, a contradiction. An argument of iteration allows us to construct the families $\left\{V^{j}\right\}_{j=1}^{\infty}$ and $\left\{x_{n}^{j}\right\}_{j=1}^{\infty}$ satisfying the claims above. Furthermore, the convergence of the series $\sum_{j=1}^{\infty}\left\|V^{j}\right\|_{\dot{H}^{s_{c}}}^{2}+$ $\left\|V^{j}\right\|_{\dot{H}^{1}}^{2}$ implies that $\left\|V^{j}\right\|_{\dot{H}^{s_{c}}}^{2}+\left\|V^{j}\right\|_{\dot{H}^{1}}^{2} \rightarrow 0$ as $j \rightarrow \infty$. By construction, $\eta\left(v_{n}^{j}\right) \leq$ $2\left(\left\|V^{j}\right\|_{\dot{H}^{s_{c}}}^{2}+\left\|V^{j}\right\|_{\dot{H}^{1}}^{2}\right)^{\frac{1}{2}} \rightarrow 0$ as $j \rightarrow \infty$, which implies (3.4).

To complete the proof of Proposition 3.1, it suffices to show $\lim \sup _{n \rightarrow \infty}\left\|v_{n}^{l}\right\|_{r} \rightarrow$ 0 as $l \rightarrow \infty$ for every $r \in\left(p_{c}, 2^{*}\right)$. For that purpose, we introduce $\mathcal{X}_{R} \in \mathcal{S}\left(\mathbb{R}^{N}\right)$ satisfying

$$
\hat{\mathcal{X}}_{R}(\xi)=1 \quad \text { if } \quad \frac{1}{R} \leq|\xi| \leq R ; \quad \hat{\mathcal{X}}_{R}(\xi)=0 \quad \text { if } \quad|\xi| \geq 2 R \quad \text { or } \quad|\xi| \leq \frac{1}{2 R} .
$$

By Fourier transform, we have $v_{n}^{l}=\mathcal{X}_{R} * v_{n}^{l}+\left(\delta-\mathcal{X}_{R}\right) * v_{n}^{l}$, where $*$ stands for the convolution and $\delta$ for the Dirac distribution. Using the Sobolev embedding $\dot{H}^{s_{c}} \hookrightarrow L^{p_{c}}$, we have

$$
\begin{aligned}
\left\|\left(\delta-\mathcal{X}_{R}\right) * v_{n}^{l}\right\|_{p_{c}} & \leq C\left\|\left(\delta-\mathcal{X}_{R}\right) * v_{n}^{l}\right\|_{\dot{H}^{s_{c}}} \\
& \leq C\left(\int|\xi|^{2 s_{c}}\left|\left(1-\hat{\mathcal{X}}_{R}(\xi)\right) \hat{v}_{n}^{l}\right|^{2} d \xi\right)^{\frac{1}{2}} \\
& \leq C\left(\int_{|\xi| \leq \frac{1}{R}}|\xi|^{2 s_{c}}\left|\hat{v}_{n}^{l}(\xi)\right|^{2} d \xi+\int_{|\xi| \geq R}|\xi|^{2 s_{c}}\left|\hat{v}_{n}^{l}(\xi)\right|^{2} d \xi\right)^{\frac{1}{2}} .
\end{aligned}
$$


Since $\int|\xi|^{2 s_{c}}\left|\hat{v}_{n}^{l}(\xi)\right|^{2} d \xi=\left\|v_{n}^{l}\right\|_{\dot{H}^{s_{c}}}^{2}$ is uniformly bounded, then

$$
\limsup _{n \rightarrow \infty} \int_{|\xi| \leq \frac{1}{R}}|\xi|^{2 s_{c}}\left|\hat{v}_{n}^{l}(\xi)\right|^{2} d \xi+\int_{|\xi| \geq R}|\xi|^{2 s_{c}}\left|\hat{v}_{n}^{l}(\xi)\right|^{2} d \xi \rightarrow 0 \quad \text { as } \quad R \rightarrow \infty .
$$

Using the Sobolev embedding $\dot{H}^{1} \hookrightarrow L^{2^{*}}$, we have

$$
\begin{aligned}
\left\|\left(\delta-\mathcal{X}_{R}\right) * v_{n}^{l}\right\|_{2^{*}} & \leq C\left\|\left(\delta-\mathcal{X}_{R}\right) * v_{n}^{l}\right\|_{\dot{H}^{1}} \leq C\left(\int|\xi|^{2}\left|\left(1-\hat{\mathcal{X}}_{R}(\xi)\right) \hat{v}_{n}^{l}\right|^{2} d \xi\right)^{\frac{1}{2}} \\
& \leq C\left(\int_{|\xi| \leq \frac{1}{R}}|\xi|^{2}\left|\hat{v}_{n}^{l}(\xi)\right|^{2} d \xi+\int_{|\xi| \geq R}|\xi|^{2}\left|\hat{v}_{n}^{l}(\xi)\right|^{2} d \xi\right)^{\frac{1}{2}} .
\end{aligned}
$$

Since $\left\|v_{n}^{l}\right\|_{\dot{H}^{1}}^{2}$ is uniformly bounded, then

$$
\limsup _{n \rightarrow \infty} \int_{|\xi| \leq \frac{1}{R}}|\xi|^{2}\left|\hat{v}_{n}^{l}(\xi)\right|^{2} d \xi+\int_{|\xi| \geq R}|\xi|^{2}\left|\hat{v}_{n}^{l}(\xi)\right|^{2} d \xi \rightarrow 0 \quad \text { as } \quad R \rightarrow \infty .
$$

By Hölder inequalities,

$$
\begin{aligned}
& \limsup _{n \rightarrow \infty}\left\|\left(\delta-\mathcal{X}_{R}\right) * v_{n}^{l}\right\|_{r} \\
& \quad \leq \limsup _{n \rightarrow \infty}\left\|\left(\delta-\mathcal{X}_{R}\right) * v_{n}^{l}\right\|_{2^{*}}^{1-\theta}\left\|\left(\delta-\mathcal{X}_{R}\right) * v_{n}^{l}\right\|_{p_{c}}^{\theta} \rightarrow 0 \quad \text { as } \quad R \rightarrow \infty
\end{aligned}
$$

where $r \in\left(p_{c}, 2^{*}\right)$, satisfying $\frac{1}{r}=\frac{\theta}{p_{c}}+\frac{1-\theta}{2^{*}}$ with $0<\theta<1$.

On the other hand, one can estimate

$$
\left\|\mathcal{X}_{R} * v_{n}^{l}\right\|_{r} \leq C\left\|\mathcal{X}_{R} * v_{n}^{l}\right\|_{\infty^{r}}^{\frac{r-p_{c}}{r}}\left\|\mathcal{X}_{R} * v_{n}^{l}\right\|_{p_{c}}^{\frac{p_{c}}{r}} \leq C\left\|\mathcal{X}_{R} * v_{n}^{l}\right\|_{\infty^{r}}^{\frac{r-p_{c}}{r}}\left\|\mathcal{X}_{R} * v_{n}^{l}\right\|_{\dot{H}^{s_{c}}}^{\frac{p_{c}}{r}} .
$$

Observe that $\lim \sup _{n \rightarrow \infty}\left\|\mathcal{X}_{R} * v_{n}^{l}\right\|_{\infty}=\sup _{\left\{x_{n}\right\}} \lim \sup _{n \rightarrow \infty}\left|\mathcal{X}_{R} * v_{n}^{l}\left(x_{n}\right)\right|$. So by definition of $\mathcal{V}\left(v_{n}^{l}\right)$, we infer

$$
\limsup _{n \rightarrow \infty}\left\|\mathcal{X}_{R} * v_{n}^{l}\right\|_{\infty} \leq \sup \left\{\int \mathcal{X}_{R}(-x) V(x) d x: V \in \mathcal{V}\left(v_{n}^{l}\right)\right\}
$$

By Parseval identity and Hölder's inequality, we have

$$
\begin{aligned}
\left|\int \mathcal{X}_{R}(-x) V(x) d x\right| & =\left|\int \mathcal{F}^{-1}\left[\mathcal{X}_{R}(-x)\right] \mathcal{F}(V) d \xi\right| \\
& \leq C \int_{\frac{1}{2 R} \leq|\xi| \leq 2 R} \frac{1}{|\xi|}|\xi||\hat{V}(\xi)| d \xi \leq C R^{\frac{N}{2}+1}\|\nabla V\|_{2} \leq C R^{\frac{N}{2}+1} \eta\left(v_{n}^{l}\right),
\end{aligned}
$$

where $\mathcal{F}$ and $\mathcal{F}^{-1}$ stand for the Fourier transform and its inverse transform respectively. Since $v_{n}^{l}$ are uniformly bounded in $\dot{H}^{s_{c}} \cap \dot{H}^{1}$, we obtain from (3.7) that

$$
\limsup _{n \rightarrow \infty}\left\|\mathcal{X}_{R} * v_{n}^{l}\right\|_{r} \leq C(R) \eta\left(v_{n}^{l}\right)^{1-\frac{p_{c}}{r}} .
$$

Since $\limsup _{n \rightarrow \infty}\left\|v_{n}^{l}\right\|_{r} \leq \lim \sup _{n \rightarrow \infty}\left(\left\|\mathcal{X}_{R} * v_{n}^{l}\right\|_{r}+\left\|\left(\delta-\mathcal{X}_{R}\right) * v_{n}^{l}\right\|_{r}\right)$, then from (3.6), (3.8) and $\eta\left(v_{n}^{l}\right) \rightarrow 0$ as $l \rightarrow \infty$, we successively let $l$ and $R$ go to infinity to obtain finally that for every $p_{c}<r<2^{*}, \limsup _{n \rightarrow \infty}\left\|v_{n}^{l}\right\|_{r} \rightarrow 0$ as $l \rightarrow \infty$, concluding the proof of Proposition 3.1 .

Theorem 1.3 is a consequence of the following proposition. 
Proposition 3.2. Let $\left\{v_{n}\right\}_{n=1}^{\infty}$ be a bounded family of $\dot{H}^{s_{c}} \cap \dot{H}^{1}$ such that

$$
\limsup _{n \rightarrow \infty}\left\|\nabla v_{n}\right\|_{2} \leq M, \quad \limsup _{n \rightarrow \infty}\left\|v_{n}\right\|_{p+1} \geq m>0 .
$$

Then, there exists $\left\{x_{n}\right\}_{n=1}^{\infty} \in \mathbb{R}^{N}$ such that, up to a subsequence,

$$
v_{n}\left(\cdot+x_{n}\right) \rightarrow V \quad \text { weakly in } \dot{H}^{s_{c}} \cap \dot{H}^{1}
$$

with

$$
\|V\|_{\dot{H}^{s_{c}}}^{p-1} \geq \frac{2 m^{p+1}}{(p+1) M^{2}}\|\tilde{Q}\|_{\dot{H}^{s_{c}}}^{p-1},
$$

with the same $\tilde{Q}$ as that in Theorem 1.3 .

We do not prove Proposition 3.2 here but will show a very similar proof (Theorem 3.3 later, since they both can be proved with the same method. Now we prove Theorem 1.3 using the above two propositions.

Proof of Theorem 1.3. Let $\left\{t_{n}\right\}_{n=1}^{\infty}$ be an arbitrary time sequence such that $t_{n} \uparrow$ $T_{+}$. Set

$$
\rho_{n}=\frac{\|\nabla \tilde{Q}\|_{2}^{\frac{1}{1-s_{c}}}}{\left\|\nabla u\left(t_{n}\right)\right\|_{2}^{\frac{1}{1-s_{c}}}} \quad \text { and } \quad v_{n}=\rho_{n}^{\frac{2}{p-1}} u\left(t_{n}, \rho_{n} x\right) .
$$

Then, by assumption (1.3), $v_{n}$ satisfies that $\left\|v_{n}\right\|_{\dot{H}^{s_{c}}}=\left\|u\left(t_{n}\right)\right\|_{\dot{H}^{s_{c}}}<\infty$ uniformly in $n$. Moreover, we get

$$
\left\|\nabla v_{n}\right\|_{2}=\rho_{n}^{1-s_{c}}\left\|\nabla u\left(t_{n}\right)\right\|_{2}=\|\nabla \tilde{Q}\|_{2}
$$

and

$$
E\left(v_{n}\right)=\rho_{n}^{2\left(1-s_{c}\right)} E\left(u\left(t_{n}\right)\right)=\rho_{n}^{2\left(1-s_{c}\right)} E\left(u_{0}\right) .
$$

From the blowup criteria established in Proposition [1.2, we get that $\rho_{n} \rightarrow 0$ and thus $E\left(v_{n}\right) \rightarrow 0$ as $n \rightarrow \infty$, which implies that $\left\|v_{n}\right\|_{p+1}^{p+1} \rightarrow \frac{p+1}{2}\|\nabla \tilde{Q}\|_{2}^{2}$.

If we set $m=\left(\frac{p+1}{2}\|\nabla \tilde{Q}\|_{2}^{2}\right)^{\frac{1}{p+1}}$ and $M=\|\nabla \tilde{Q}\|_{2}$, applying Proposition 3.2 to $\left\{v_{n}\right\}_{n=1}^{\infty}$, which is a bounded sequence in $\dot{H}^{s_{c}} \cap \dot{H}^{1}$ by construction, we obtain a family $\left\{x_{n}\right\}_{n=1}^{\infty}$ and a profile $V$ with

$$
\|V\|_{\dot{H}^{s_{c}}}^{p-1} \geq \frac{2 m^{p+1}}{(p+1) M^{2}}\|\tilde{Q}\|_{\dot{H}^{s_{c}}}^{p-1}=\|\tilde{Q}\|_{\dot{H}^{s_{c}}}^{p-1}
$$

such that, up to a subsequence, $\rho_{n}^{\frac{2}{p-1}} u\left(t_{n}, \rho_{n} \cdot+x_{n}\right) \rightarrow V$ weakly in $\dot{H}^{s_{c}} \cap \dot{H}^{1}$. Thus, we infer from the definition of $\dot{H}^{s}$ (see section 1) that

$$
(-\Delta)^{\frac{s_{c}}{2}} \rho_{n}^{\frac{2}{p-1}} u\left(t_{n}, \rho_{n} \cdot+x_{n}\right) \rightarrow(-\Delta)^{\frac{s_{c}}{2}} V \text { weakly in } L^{2} .
$$

Consequently, for every $R>0$,

$$
\int_{|x| \leq R}\left|(-\Delta)^{\frac{s_{c}}{2}} V(x)\right|^{2} d x \leq \liminf _{n \rightarrow \infty} \int_{\left|x-x_{n}\right| \leq \rho_{n} R}\left|(-\Delta)^{\frac{s_{c}}{2}} u\left(t_{n}, x\right)\right|^{2} d x .
$$

Assumption (1.4) implies that $\frac{\lambda\left(t_{n}\right)}{\rho_{n}} \rightarrow \infty$ as $n \rightarrow \infty$, which immediately gives

$$
\int\left|(-\Delta)^{\frac{s_{c}}{2}} V(x)\right|^{2} d x \leq \liminf _{n \rightarrow \infty} \sup _{y \in \mathbb{R}^{N}} \int_{|x-y| \leq \lambda\left(t_{n}\right)}\left|(-\Delta)^{\frac{s_{c}}{2}} u\left(t_{n}, x\right)\right|^{2} d x .
$$


Since the sequence $\left\{t_{n}\right\}_{n=1}^{\infty}$ is arbitrary, we infer that

$$
\int\left|(-\Delta)^{\frac{s_{c}}{2}} V(x)\right|^{2} d x \leq \liminf _{t \rightarrow T_{+}} \sup _{y \in \mathbb{R}^{N}} \int_{|x-y| \leq \lambda(t)}\left|(-\Delta)^{\frac{s_{c}}{2}} u(t, x)\right|^{2} d x .
$$

For every fixed $t \in\left[0, T_{+}\right)$, the function $y \mapsto \int_{|x-y| \leq \lambda(t)}\left|(-\Delta)^{\frac{s_{c}}{2}} u(t, x)\right|^{2} d x$ is continuous and goes to zero at infinity. Thus, there exists $x(t) \in \mathbb{R}^{N}$ such that

$$
\int_{|x-x(t)| \leq \lambda(t)}\left|(-\Delta)^{\frac{s_{c}}{2}} u(t, x)\right|^{2} d x=\sup _{y \in \mathbb{R}^{N}} \int_{|x-y| \leq \lambda(t)}\left|(-\Delta)^{\frac{s_{c}}{2}} u(t, x)\right|^{2} d x,
$$

which, together with (3.11), immediately gives (1.5) and completes the proof.

In order to show Theorem 1.4 we should first prove the following theorem.

Theorem 3.3. Let $\left\{v_{n}\right\}_{n=1}^{\infty}$ be a bounded family of $\dot{H}^{s_{c}} \cap \dot{H}^{1}$ such that

$$
\limsup _{n \rightarrow \infty}\left\|\nabla v_{n}\right\|_{2} \leq M, \quad \limsup _{n \rightarrow \infty}\left\|v_{n}\right\|_{p+1} \geq m>0 .
$$

Then, there exists $\left\{x_{n}\right\}_{n=1}^{\infty} \in \mathbb{R}^{N}$ such that, up to a subsequence, $v_{n}\left(\cdot+x_{n}\right) \rightarrow V$ weakly in $\dot{H}^{s_{c}} \cap \dot{H}^{1}$ with

$$
\|V\|_{p_{c}}^{p-1} \geq \frac{2 m^{p+1}}{(p+1) M^{2}}\left\|Q^{\prime}\right\|_{p_{c}}^{p-1}
$$

where we use the same $Q^{\prime}$ as in Theorem 1.4 .

Proof. Applying Proposition 3.1 to the sequence $\left\{v_{n}\right\}_{n=1}^{\infty}$, we obtain the decomposition $v_{n}(x)=\sum_{j=1}^{l} V^{j}\left(x-x_{n}^{j}\right)+v_{n}^{l}(x)$ such that (3.3) holds. Since $p_{c}=\frac{2 N}{N-2 s_{c}}<$ $\frac{2 N+2\left(1-s_{c}\right)}{N-2 s_{c}}=p+1<2^{*}$, we get, in particular,

$$
m^{p+1} \leq \limsup _{n \rightarrow \infty}\left\|\sum_{j=1}^{\infty} V^{j}\left(x-x_{n}^{j}\right)\right\|_{p+1}^{p+1} .
$$

From the elementary inequality

$$
\left.|| \sum_{j=1}^{l} a_{j}\right|^{p+1}-\left.\sum_{j=1}^{l}\left|a_{j}\right|^{p+1}\left|\leq C \sum_{k \neq j}\right| a_{j}|| a_{k}\right|^{p}
$$

we get that

$$
\begin{aligned}
& \int\left|\sum_{j=1}^{l} V^{j}\left(x-x_{n}^{j}\right)\right|^{p+1} d x \\
& \quad \leq \sum_{j=1}^{l} \int\left|V^{j}\left(x-x_{n}^{j}\right)\right|^{p+1} d x+C \sum_{j \neq k} \int\left|V^{j}\left(x-x_{n}^{j}\right)\right|^{p}\left|V^{k}\left(x-x_{n}^{k}\right)\right| d x \\
& \quad=\sum_{j=1}^{l} \int\left|V^{j}\left(x-x_{n}^{j}\right)\right|^{p+1} d x+C \sum_{j \neq k} \int\left|V^{j}(x)\right|^{p} \mid V^{k}\left(x+\left(x_{n}^{j}-x_{n}^{k}\right) \mid d x .\right.
\end{aligned}
$$

From (3.1), $\left|x_{n}^{j}-x_{n}^{k}\right| \rightarrow \infty$ as $n \rightarrow \infty$ for $j \neq k$. Thus from the Hölder inequalities, we get that $V^{k}\left(\cdot+\left(x_{n}^{j}-x_{n}^{k}\right) \rightarrow 0\right.$ for any $j \neq k$ and that the mixed terms in the sum of (3.12) tend to vanish as $n \rightarrow \infty$. Thus, we obtain 
$m^{p+1} \leq \lim \sup _{n \rightarrow \infty} \sum_{j=1}^{\infty}\left\|V^{j}\right\|_{p+1}^{p+1}$. On the other hand, in view of the GagliardoNirenberg inequality (2.2), we obtain that

$$
\sum_{j=1}^{\infty}\left\|V^{j}\right\|_{p+1}^{p+1} \leq c_{p_{c}} \sum_{j=1}^{\infty}\left\|V^{j}\right\|_{p_{c}}^{p-1}\left\|\nabla V^{j}\right\|_{2}^{2} \leq c_{p_{c}} \sup _{j \geq 1}\left\|V^{j}\right\|_{p_{c}}^{p-1} \sum_{j=1}^{\infty}\left\|\nabla V^{j}\right\|_{2}^{2} .
$$

From (3.3), we get $\sum_{j=1}^{\infty}\left\|\nabla V^{j}\right\|_{2}^{2} \leq \lim \sup _{n \rightarrow \infty}\left\|\nabla v_{n}\right\|_{2}^{2} \leq M^{2}$. Therefore,

$$
\sup _{j \geq 1}\left\|V^{j}\right\|_{p_{c}}^{p-1} \geq \frac{m^{p+1}}{c_{p_{c}} M^{2}}
$$

By the Sobolev imbedding $\dot{H}^{s_{c}} \hookrightarrow L^{p_{c}}$ and in view of the convergence of the series $\sum_{j=1}^{\infty}\left\|V^{j}\right\|_{\dot{H}^{s_{c}}}^{2}$, we get that $\sum_{j=1}^{\infty}\left\|V^{j}\right\|_{p_{c}}^{2}<\infty$. This implies that there exist some $j_{0} \geq 1$ such that $\left\|V^{j_{0}}\right\|_{p_{c}}=\sup _{j \geq 1}\left\|V^{j}\right\|_{p_{c}}$. By Theorem 2.1, we have that $c_{p_{c}}=\frac{p+1}{2}\left\|Q^{\prime}\right\|_{p_{c}}^{-(p-1)}$. Thus, (3.14) implies that

$$
\left\|V^{j_{0}}\right\|_{p_{c}}^{p-1} \geq \frac{2 m^{p+1}}{(p+1) M^{2}}\left\|Q^{\prime}\right\|_{p_{c}}^{p-1} .
$$

Now, by a change of variables, (3.2) gives that

$$
v_{n}\left(x+x_{n}^{j_{0}}\right)=V^{j_{0}}(x)+\sum_{j \neq j_{0}} V^{j}\left(x+x_{n}^{j_{0}}-x_{n}^{j}\right)+\tilde{v}_{n}^{l}(x),
$$

where $\tilde{v}_{n}^{l}(x)=v_{n}^{l}\left(x+x_{n}^{j_{0}}\right)$. As discussed above, $\left|x_{n}^{j_{0}}-x_{n}^{j}\right| \rightarrow \infty$ as $n \rightarrow \infty$ implies that

$$
V^{j}\left(\cdot+x_{n}^{j_{0}}-x_{n}^{j}\right) \rightarrow 0 \quad \text { weakly in } \quad \dot{H}^{s_{c}} \cap \dot{H}^{1} \quad \text { for every } j \neq j_{0} .
$$

Hence, we obtain that $v_{n}\left(\cdot+x_{n}^{j_{0}}\right) \rightarrow V^{j_{0}}+v^{l}$ weakly in $\dot{H}^{s_{c}} \cap \dot{H}^{1}$, where $v^{l}$ denotes the weak limit of $\left\{\tilde{v}_{n}^{l}\right\}_{n=1}^{\infty}$. However, since

$$
\left\|v^{l}\right\|_{p+1} \leq \limsup _{n \rightarrow \infty}\left\|\tilde{v}_{n}^{l}\right\|_{p+1}=\limsup _{n \rightarrow \infty}\left\|v_{n}^{l}\right\|_{p+1} \rightarrow 0, \quad l \rightarrow \infty,
$$

the uniqueness of the weak limit implies that $v^{l}=0$ for every $l \geq j_{0}$. Thus, $v_{n}\left(\cdot+x_{n}^{j_{0}}\right) \rightarrow V^{j_{0}}$ weakly in $\dot{H}^{s_{c}} \cap \dot{H}^{1}$. Then the sequence $\left\{x_{n}^{j_{0}}\right\}_{n=1}^{\infty}$ and the function $V^{j}$ fulfill the conditions of Theorem 3.3 .

Remark 3.4. From the proof above, we can also replace $c_{p_{c}}$ in (3.13) with $c_{N}^{2}=$ $\|\nabla W\|_{2}^{-\frac{4}{N}}$ (see section 2 and Remark 2.4), and by the same argument, we should then obtain that the weak limit $V$ satisfies that

$$
\|V\|_{p_{c}}^{p-1} \geq \frac{m^{p+1}}{M^{2}}\|\nabla W\|_{2}^{\frac{4}{N}} .
$$

Now we sketch the proof of Theorem 1.4 by the same method as used in the proof of Theorem 1.3 .

Proof of Theorem 1.4. Let $\left\{t_{n}\right\}_{n=1}^{\infty}$ be an arbitrary time sequence such that $t_{n} \uparrow$ $T_{+}$. Set $\rho_{n}=\left\|\nabla Q^{\prime}\right\|_{2}^{\frac{1}{1-s_{c}}} /\left\|\nabla u\left(t_{n}\right)\right\|_{2}^{\frac{1}{1-s_{c}}}$ and $v_{n}=\rho_{n}^{\frac{2}{p-1}} u\left(t_{n}, \rho_{n} x\right)$. Similarly to the proof of Theorem 1.3, we obtain

$$
\left\|v_{n}\right\|_{\dot{H}^{s_{c}}}=\left\|u\left(t_{n}\right)\right\|_{\dot{H}^{s_{c}}}<\infty, \quad\left\|\nabla v_{n}\right\|_{2}=\left\|\nabla Q^{\prime}\right\|_{2}, \quad \text { and } \quad E\left(v_{n}\right)=\rho_{n}^{2\left(1-s_{c}\right)} E\left(u_{0}\right) .
$$


Moreover, $\left\|v_{n}\right\|_{p+1}^{p+1} \rightarrow \frac{p+1}{2}\left\|\nabla Q^{\prime}\right\|_{2}^{2}$. Applying Theorem [3.3 to $\left\{v_{n}\right\}_{n=1}^{\infty}$ with $m=$ $\left(\frac{p+1}{2}\left\|\nabla Q^{\prime}\right\|_{2}^{2}\right)^{\frac{1}{p+1}}$ and $M=\left\|\nabla Q^{\prime}\right\|_{2}$, we obtain a family $\left\{x_{n}\right\}_{n=1}^{\infty}$ and a profile $V$ with

$$
\|V\|_{p_{c}}^{p-1} \geq \frac{2 m^{p+1}}{(p+1) M^{2}}\left\|Q^{\prime}\right\|_{p_{c}}^{p-1}=\left\|Q^{\prime}\right\|_{p_{c}}^{p-1}
$$

such that, up to a subsequence, $\rho_{n}^{\frac{2}{p-1}} u\left(t_{n}, \rho_{n} \cdot+x_{n}\right) \rightarrow V$ weakly in $\dot{H}^{s_{c}} \cap \dot{H}^{1}$. Consequently, for every $R>0$,

$$
\int_{|x| \leq R}|V(x)|^{p_{c}} d x \leq \liminf _{n \rightarrow \infty} \int_{\left|x-x_{n}\right| \leq \rho_{n} R}\left|u\left(t_{n}, x\right)\right|^{p_{c}} d x
$$

In view of assumption (1.4), by the same steps used in the proof of Theorem 1.3. we finally obtain that

$$
\|V\|_{p_{c}}^{p_{c}} \leq \liminf _{t \rightarrow T_{+}} \int_{|x-x(t)| \leq \lambda(t)}|u(t, x)|^{p_{c}} d x
$$

which together with (3.17) gives (1.7), concluding the proof.

Remark 3.5. In view of Remark 2.4 and Remark 3.4, we can obtain another form of concentration as follows:

Theorem 3.6. Let $u_{0} \in \dot{H}^{s_{c}} \cap \dot{H}^{1}$ be such that the corresponding solution $u$ to (1.1) blows up in finite time $T_{+}>0$ satisfying (1.3). Assume $\lambda(t)>0$ satisfying (1.4). Then there exists $x(t) \in \mathbb{R}^{N}$ such that

$$
\liminf _{t \rightarrow T_{+}}\|u\|_{L^{p_{c}}(|x-x(t)| \leq \lambda(t))}^{p_{c}} \geq\left(\frac{p+1}{2}\right)^{\frac{N}{2}}\|\nabla W\|_{2}^{2},
$$

where $W$ is the solution to (2.4).

In fact, to prove Theorem 3.6 , we just need to take $\rho_{n}=\frac{\|\nabla W\|_{2}^{\frac{1}{1-s_{c}}}}{\left\|\nabla u\left(t_{n}\right)\right\|_{2}^{1-s_{c}}}, m=$ $\left(\frac{p+1}{2}\|\nabla W\|_{2}^{2}\right)^{\frac{1}{p+1}}$ and $M=\|\nabla W\|_{2}$ in the proof of Theorem 1.4 and follow the argument there exactly. By Theorem 2.1 and Remark 2.4, we obtain without difficulty that $\left\|Q^{\prime}\right\|_{p_{c}}^{p_{c}} \geq\left(\frac{p+1}{2}\right)^{\frac{N}{2}}\|\nabla W\|_{2}^{2}$, which implies that the concentration property described in Theorem 1.4 should be better than that in Theorem 3.6

Note that the quantity of the least concentration described in [20] for the $L^{p_{c}}$ norm of the radial blowup solutions to (1.1) was indeed in accordance with that in Theorem 3.6. In this regard, our result obtained in Theorem 1.4 not only removes the radially symmetric assumption in the case that (1.3) holds but also quantitatively improves the concentration property obtained by [20].

\section{Remarks on Divergence of SOlUtions in $H^{1}$}

Recall that in [7, the author proved the following result.

Proposition 4.1. Suppose $u_{0} \in H^{1}, M(u)^{\frac{1-s_{c}}{s_{c}}} E(u)<M(Q)^{\frac{1-s_{c}}{s_{c}}} E(Q)$ and

$$
\left\|\nabla u_{0}\right\|_{2}\left\|u_{0}\right\|_{2}^{\frac{1-s_{c}}{s_{c}}}>\|\nabla Q\|_{2}\|Q\|_{2}^{\frac{1-s_{c}}{s_{c}}} \text {. }
$$

Then either $u(t)$ blows up in finite forward time $T_{+}>0$ or $u(t)$ is forward global and there exists a time sequence $t_{n} \rightarrow \infty$ such that $\left\|\nabla u\left(t_{n}\right)\right\|_{2} \rightarrow \infty$ as $n \rightarrow+\infty$. A similar statement holds for negative time. 
Since $u_{0} \in H^{1} \subset \dot{H}^{s_{c}} \cap \dot{H}^{1}$, if the corresponding finite time blowup solution $u(t)$ to (1.1) satisfies (1.3), then the concentration properties (1.5) and (1.7) hold by Theorem 1.3 and Theorem 1.4. On the other hand, if $T_{+}=\infty$ with the same $T_{+}$as in Proposition 1.2 and the assumptions of Proposition 4.1 hold, then we, furthermore, have the following result.

Theorem 4.2. Suppose $u_{0} \in H^{1}, M(u)^{\frac{1-s_{c}}{s_{c}}} E(u)<M(Q)^{\frac{1-s_{c}}{s_{c}}} E(Q)$ and

$$
\left\|\nabla u_{0}\right\|_{2}\left\|u_{0}\right\|_{2}^{\frac{1-s_{c}}{s_{c}}}>\|\nabla Q\|_{2}\|Q\|_{2}^{\frac{1-s_{c}}{s_{c}}} .
$$

If the corresponding solution $u(t)$ to (1.1) exists globally in positive time and satisfies (1.3) with $T_{+}=\infty$, then there exists some time sequence $t_{n} \rightarrow+\infty$ as $n \rightarrow+\infty$ and $x_{n} \in \mathbb{R}^{N}$ such that for any sequence $R_{n} \in(0,+\infty)$ satisfying

$$
R_{n}\left\|\nabla u\left(t_{n}\right)\right\|_{2}^{\frac{1}{1-s_{c}}} \rightarrow+\infty, \quad n \rightarrow+\infty
$$

the following hold:

$$
\liminf _{n \rightarrow+\infty} \int_{\left|x-x_{n}\right| \leq R_{n}}\left|(-\Delta)^{\frac{s_{c}}{2}} u\left(t_{n}, x\right)\right|^{2} d x \geq\|\tilde{Q}\|_{\dot{H}^{s_{c}}}^{2}
$$

and

$$
\liminf _{n \rightarrow+\infty} \int_{\left|x-x_{n}\right| \leq R_{n}}\left|u\left(t_{n}, x\right)\right|^{p_{c}} d x \geq\left\|Q^{\prime}\right\|_{p_{c}}^{p_{c}},
$$

where $\tilde{Q}$ and $Q^{\prime}$ are the same as in Theorem 1.3 and Theorem 1.4 .

Since the proof is similar to that for Theorem 1.3 and Theorem 1.4 we just sketch it as follows. In view of Proposition 4.1, we have obtained a time sequence $t_{n} \rightarrow+\infty$ such that $\left\|\nabla u\left(t_{n}\right)\right\|_{2} \rightarrow \infty$ as $n \rightarrow+\infty$. Using such a sequence $u\left(t_{n}\right)$, we can construct $\rho_{n} \in \mathbb{R}^{+} \backslash\{0\}$ and a bounded sequence $v_{n}$ in $\dot{H}^{s_{c}} \cap \dot{H}^{1}$, the same as in the proof of Theorem 1.3 and Theorem 1.4. Correspondingly, we then get a profile $V$ with $\|V\|_{\dot{H}^{s_{c}}} \geq\|\tilde{Q}\|_{\dot{H}^{s_{c}}}$ and some sequence $\left\{x_{n}\right\}_{n \geq 1} \subset \mathbb{R}^{N}$ such that $\rho_{n}^{\frac{2}{p-1}} u\left(t_{n}, \rho_{n} \cdot+x_{n}\right) \rightarrow V$ weakly in $\dot{H}^{s_{c}} \cap \dot{H}^{1}$. By assumption (4.1), we can finally obtain that

$$
\liminf _{n \rightarrow+\infty} \int_{\left|x-x_{n}\right| \leq R_{n}}\left|(-\Delta)^{\frac{s_{c}}{2}} u\left(t_{n}, x\right)\right|^{2} d x \geq\|\tilde{Q}\|_{\dot{H}^{s_{c}}}^{2},
$$

which is (4.2). The concentration (4.3) can be obtained similarly.

\section{REFERENCES}

[1] T. Aubin, Équations différentielles non linéaires et problème de Yamabe concernant la courbure scalaire. J. Math. Pures Appl., IX. Sér. 55 (1976), 269-296. MR0431287 (55:4288)

[2] H. Berestycki and P.L. Lions, Nonlinear scalar field equations. Arch. Ration. Mech. Anal. 82 (1983) 313-375. MR695535(84h:35054a)

[3] T. Cazenave, Semilinear Schrödinger equations. Courant Lecture Notes in Mathematics, 10. New York University, Courant Institute of Mathematical Sciences, New York; American Mathematical Society, Providence, RI, 2003. MR2002047 (2004j:35266)

[4] T. Cazenave and F. B. Weissler, Some remarks on the nonlinear Schrödinger equation in the critical case. Nonlinear semigroups, partial differential equations and attractors (Washington, DC, 1987), Lecture Notes in Math., vol. 1394, Springer-Verlag, Berlin, 1989, 18-29. MR.1021011 (91a:35149)

[5] T. Duyckaerts, J. Holmer and S. Roudenko, Scattering for the non-radial 3D cubic nonlinear Schrödinger equation, Math. Res. Letters 15 (2008), 1233-1250. MR2470397 (2010e:35257) 
[6] J. Ginibre and G. Velo, On a class of nonlinear Schrödinger equations. I. The Cauchy problem, general case; II. Scattering theory, general case, J. Func. Anal. 32 (1979), 1-32, 33-71. MR.533218 (82c:35057), MR.533219 (82c:35058)

[7] Q. Guo, Divergent solutions to the $L^{2}$-supercritical NLS equations, arXiv:1101.2271, to appear in Acta Math. Appl. Sci.

[8] J. Holmer and S. Roudenko, A sharp condition for scattering of the radial 3D cubic nonlinear Schrödinger equation, Comm. Math. Phys. 282 (2008), no. 2, 435-467. MR2421484 (2009h:35403)

[9] J. Holmer and S. Roudenko, On blow-up solutions to the 3D cubic nonlinear Schrödinger equation, Appl. Math. Anal. 38 (2006), 1035-1047. MR2354447(2008i:35227)

[10] J. Holmer and S. Roudenko, Divergence of infinite-variance nonradial solutions to the 3D NLS equation, Comm. PDE 35 (2010), 878-905. MR2753623 (2011m:35353)

[11] T. Hmidi and S. Keraani, Blowup theory for the critical nonlinear Schrödinger equations revisited, IMRN, 46, 2815-2828 (2005). MR2180464 (2007k:35464)

[12] C. E. Kenig and F. Merle, Global well-posedness, scattering, and blow-up for the energycritical, focusing, non-linear Schrödinger equation in the radial case, Invent. Math. 166 (2006), no. 3, 645-675. MR2257393 (2007g:35232)

[13] R. Killip and M. Visan, The focusing energy-critical nonlinear Schrödinger equation in dimensions five and higher, Amer J. Math. 132 (2010), no. 2, 361-424. MR.2654778 (2011e:35357)

[14] F. Merle and Y. Tsutsumi, $L^{2}$ concentration of blow-up solutions for the nonlinear Schrödinger equation with critical power nonlinearity, J.D.E. 84 (1990), no. 2, 205-214. MR.1047566 (91e:35194)

[15] C. Sulem and P. L. Sulem, The nonlinear Schrödinger equation. Self-focusing and wave collapse, Applied Mathematical Sciences, vol. 139, Springer, New York, 1999. MR.1696311 (2000f:35139)

[16] G. Talenti, Best constant in Sobolev inequality. Ann. Mat. Pura Appl. 110 (1976), 353-372. MR0463908 (57:3846)

[17] Y. Tsutsumi, Rate of $L^{2}$ concentration of blow-up solutions for the nonlinear Schrödinger equation with critical power, Nonlinear Anal. 15 (1990), no. 8, 719-724. MR 1074950 $(91 \mathrm{j}: 35043)$

[18] M. Weinstein, Nonlinear Schrödinger equations and sharp interpolation estimates, Comm. Math. Phys. 87 (1982/83), no. 4, 567-576. MR691044(84d:35140)

[19] J. Yuan, Some research on nonlinear Schrödinger equation and related problems, Ph.D. thesis (in Chinese), Chinese Academy of Mathematics and System Science (2010).

[20] S. Zhu, Dynamical properties of blow-up solutions to nonlinear Schrödinger equations, Ph.D. thesis (in Chinese), Sichuan University (2011).

Academy of Mathematics and Systems Science, Chinese Academy of Sciences, Beijing 100190, People's Republic of China

Current address: College of Science, Minzu University of China, Beijing 100081, People's Republic of China

E-mail address: guoqing@amss.ac.cn 